\title{
Sodium Fluoride Positron Emission Tomography/Computed Tomography from the Skull Base to Mid-thigh
}

National Cancer Institute

\section{Source}

National Cancer Institute. Sodium Fluoride Positron Emission Tomography/Computed

Tomography from the Skull Base to Mid-thigh. NCI Thesaurus. Code C137905.

Positron emission tomography combined with computed tomography of the region

encompassing the skull base to the mid-thigh with the use of the contrast agent sodium fluoride F 18 (18F-NaF) to enhance the image. 